\title{
Design and Analysis of RF MEMS Capacitive Shunt Switch and Impact of Geometric Trade-offs on RF Performance
}

\author{
${ }^{* 1}$ Surendra K. Waghmare, ${ }^{2}$ Dilip D. Shah \\ ${ }^{1}$ Research Scholar, BDCOE, Sevagram, Wardha and Faculty, E\&TC Engineering, GHRCEM, Wagholi, Pune \\ ${ }^{2}$ JSPM's Imperial College of Engineering and Research, Wagholi, Pune \\ *Email: surendra.waghmare@raisoni.net
}

Received: $22^{\text {nd }}$ December 2018, Accepted: $13^{\text {th }}$ February 2019, Published: $30^{\text {th }}$ June $_{2019}$

\begin{abstract}
The electromagnetic and the electromechanical characteristics of the radio frequency micro-electro-mechanicalsystem (RF MEMS) switches for high-frequency applications are the critical performance metrics that need to optimize. Performance indices of the RF MEMS switches such as isolation, insertion loss, pull-in voltage, holddown voltage, reliability are dependent on types and properties of conducting and insulating materials that are used in the construction of switch. This article proposes the design and analysis of the two terminal capacitive shunt switches built on a coplanar waveguide (CPW) for applications in subsets of Ka- and V-Band frequency range. The proposed switch used a fixed-fixed gold membrane with the low-spring constant uniform single meander flexures support and achieved a low pull-in voltage of 5.1 Volts. An impact of the variation of the geometric parameter trade-offs like conducting membrane height, dielectric material height, and the air gap between the membrane and the dielectric materials like Silicon Nitride $\left(\mathrm{Si}_{3} \mathrm{~N} 4\right)$ and Hafnium Dioxide $\left(\mathrm{HfO}_{2}\right)$ are studied to investigate RF and electromechanical performance of the switch.
\end{abstract}

Keywords

RF MEMS Switch, Pull-in Voltage, Geometric Trades Off's Variations, S-Parameters

\section{Introduction}

The RF MEMS switches are widely finding their use as an essential component in the RF/microwave/mm-wave devices and circuits applicable in the commercial and defense products. RF MEMS switches are favored over the semiconductor devices (PIN Diodes and MESFETs or JFETs) due to their potential performance such as high isolation, low insertion loss, broader operating bandwidth, high capacitance ratio, almost zero power consumption, 20-40 times higher cut-off frequency. The drawback of the RF MEMS switches is its higher actuation voltage which leads to use of an additional up-converters increasing device size. Also, the high electric field at the insulating layer results into charge injection or dielectric breakdown and thus limits the reliability of the switches (Tilmans, 2002; Rebeiz, 2003; M. Angira et al., 2015). While designing an electrostatic actuationbased RF MEMS switches for the high-frequency applications, it is desirable to have a low pull-in voltage for actuation and excellent RF performance for required frequency bands of operation. To optimize these performance indices, types, and properties of conducting and insulating materials play an essential role (Sharma et al., 2012).

Till date, many researchers have studied and represented the design aspects of the RF MEMS capacitive shunt switches on the geometric parameter trade-offs. A RF MEMS switch was designed and simulated (P. Bahmanyar et al. 2010) based on silicon substrate coplanar waveguide (CPW) with $\mathrm{Si}_{3} \mathrm{~N}_{4}$ insulating material and shown good RF response such as insertion loss better than $-0.65 \mathrm{~dB}$ and high isolation of $-38 \mathrm{~dB}$ in Ka-band and demonstrated the effect of the variation of the geometric parameters on switching behaviour. Simulation results show that varying the width of the membrane, it effects on the resonance frequency and isolation loss in the downstate performance of the switch. Also, decreasing the thickness of the insulating material $\left(\mathrm{Si}_{3} \mathrm{~N}_{4}\right)$ improved isolation.

Design and simulation of an RF MEMS shunt switch for Ka and V-Bands were also done (Y. Mafinejad et al. 2009). The switch design represented and discussed the impact of the geometric parameters such as membrane height, membrane overlap area and dielectric thickness on the electromagnetic behavior of the switch.

This research article focusses on the design and analysis of gold membrane low-spring constant two terminal switches with a uniform single meandered support to optimize low-pull in voltage using FEM COMSOL ${ }^{\mathrm{TM}}$ tool. The impact of the different geometric trade off's such as membrane height, dielectric height and air gap on the electromagnetic behavior of the switch are simulated with $\mathrm{HFSS}^{\mathrm{TM}}$. The dielectric materials such as $\mathrm{Si}_{3} \mathrm{~N}_{4}$ and $\mathrm{HfO}_{2}$ are used to investigate the RF Performance.

Proposed RF MEMS Switch Design

The typical two terminal step type membrane RF MEMS fixed-fixed capacitive shunt switch is shown in (Fig 1). It uses a gold metal membrane anchored at either end and electrically coupled to the ground of $50 \Omega \mathrm{CPW}$. The central conductor of the CPW carries the RF signal and also acts as a DC actuation electrode for two 
terminal switches. A thinner insulating material like $\mathrm{Si}_{3} \mathrm{~N}_{4}$ or $\mathrm{HfO}_{2}$ are coated over the central conductor under the central membrane area which forms a capacitive effect in the switch. When the switch is in the upstate, i.e., an unbiased state, the capacitance formed by the metal membrane-air-dielectric-central conductor of CPW is minimal which do not affect the impedance of the switch and allows the RF signal to pass from one end to the another and of the transmission line. When the switch is biased between the upper metal membrane and the central conductor of the $\mathrm{CPW}$, an electrostatic force pulls down the upper metal membrane on a dielectric layer above the CPW transmission line. The capacitance due to metal-dielectric-metal becomes very high which affects the impedance of the switch and couples the RF signal to ground via the metal membrane.

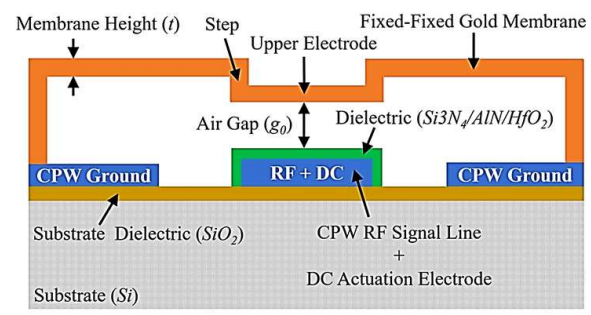

Figure 1: Step Type Membrane-Based RF MEMS Capacitive Shunt Switch

Based on two terminal switch topologies, the proposed RF MEMS switch design with the geometric dimensions and 3D-layout using HFSS ${ }^{\mathrm{TM}}$ is shown in (Fig 2) and (Fig 3) respectively.

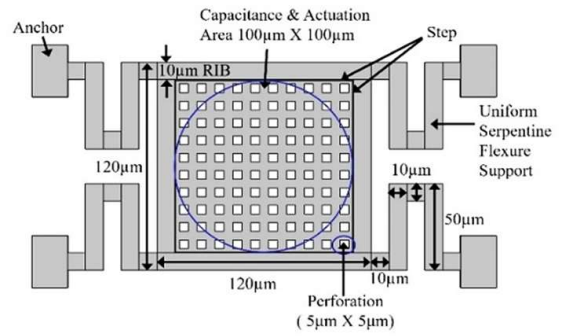

Figure 2: Membrane Dimensions of the Proposed RF MEMS Capacitive Shunt Switch

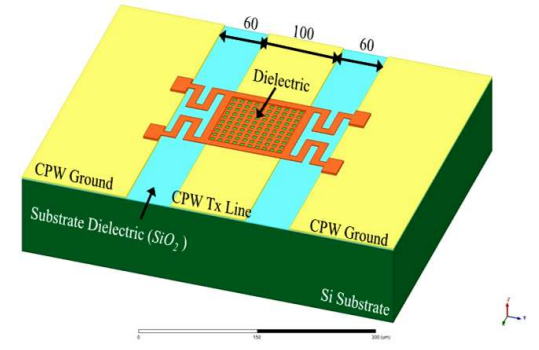

Figure 3: The 3-D Layout of the Switch in CPW Configuration

Pull-in Voltage and Switching Time Analysis for Low Spring Constant Uniform Meander Design

The switch is electrostatically driven which requires an actuation voltage $\left(\mathrm{V}_{\mathrm{P}}\right)$ to deflect the membrane by $2 \mu \mathrm{m}$ as per the Eq. (1) (Rebeiz et al., 2001).

$$
\mathrm{V}_{\mathrm{P}}=\sqrt{\frac{8 \mathrm{k}_{\mathrm{Z}} \mathrm{g}_{0}^{3}}{27{ }_{0} \mathrm{~A}}}
$$

where $\mathrm{k}_{\mathrm{Z}}$ is an effective spring constant of the structure due to the material properties such as Young's modulus E (70 GPa for Gold), a moment of inertia I $\left(\mathrm{m}^{4}\right)$, Poisson's ratio (v), internal bilateral residual stress, and the shape and the dimension of the mechanical structures. ' $A$ ' is the electrostatic actuation area of the electrodes where the electrostatic force is imparted (here $100 \mu \mathrm{m} \times 100 \mu \mathrm{m}), g_{0}$ is the zero-bias air gap $(2 \mu \mathrm{m})$ between the movable membrane and the dielectric layer above the lower electrode, and $\varepsilon_{0}$ is the free-space permittivity. Pullin voltage is dependent on $k_{Z}$ which is the overall effective spring constant of the membrane calculated as given by Eq. (2) (Rebeiz, 2003).

$$
\mathrm{k}_{\mathrm{Z}}=\frac{\mathrm{k}_{\mathrm{m}} \times \mathrm{k}_{\mathrm{n}-\mathrm{m}}}{\mathrm{k}_{\mathrm{m}}+\mathrm{k}_{\mathrm{n}-\mathrm{m}}}
$$


where $k_{m}$ is a spring constant of a meandered flexure and $k_{n-m}$ is a spring constant of non-meandered membrane expressed by Eq. (3) and Eq. (4) (Rebeiz, 2003).

$$
\begin{aligned}
& \frac{1}{\mathrm{k}_{\mathrm{m}}}=\frac{1}{\mathrm{k}_{1}}+\frac{1}{\mathrm{k}_{2}}+\frac{1}{\mathrm{k}_{3}}+\frac{1}{\mathrm{k}_{4}}+\frac{1}{\mathrm{k}_{5}} \\
& \mathrm{k}_{\mathrm{n}-\mathrm{m}}=32 \mathrm{Ew}_{\mathrm{n}-\mathrm{m}}\left(\frac{\mathrm{t}}{\mathrm{L}_{\mathrm{n}-\mathrm{m}}}\right)^{3}
\end{aligned}
$$

In Eq. (3) the spring constant of the individual arms of flexure is calculated using Eq. (5) as:

$\mathrm{k}=\operatorname{Ew}\left(\frac{\mathrm{t}}{\mathrm{L}}\right)^{3}$

Here $w_{n-m}$ and $L_{n-m}$ are the widths and the length of the non-meandered bridge.

Dimensions for the meandered flexure membrane and non-meandered membrane according to (Fig 2) are given in Table 1.

\begin{tabular}{|c|c|c|c|c|c|}
\hline \multirow{2}{*}{ Component } & \multicolumn{2}{|c|}{ Spring-Constant } & \multicolumn{3}{|c|}{ Dimensions $(\boldsymbol{\mu m})$} \\
\cline { 3 - 6 } & \multicolumn{2}{|c|}{} & $\mathbf{L}$ & $\mathbf{W}$ & $\mathbf{t}$ \\
\hline Non-Meandered Membrane & \multicolumn{2}{|c|}{$k_{n-m}$} & 120 & 120 & 0.5 \\
\hline \multirow{3}{*}{ Meandered Membrane } & \multirow{3}{*}{$k_{m}$} & $k_{1}$ & 10 & 10 & 0.5 \\
\cline { 3 - 6 } & & $k_{2}$ & 50 & 10 & 0.5 \\
\cline { 3 - 6 } & & $k_{3}$ & 10 & 10 & 0.5 \\
\cline { 3 - 6 } & & $k_{4}$ & 50 & 10 & 0.5 \\
\cline { 3 - 6 } & & $k_{5}$ & 10 & 10 & 0.5 \\
\hline
\end{tabular}

Table 1: Dimensions of Meandered and Non-Meandered Membrane

The theoretically calculated value for $k_{m}$ is found to be $0.34 \mathrm{~N} / \mathrm{m}$, and for that non-meandered membrane $k_{n-m}$, it is $19.44 \mathrm{~N} / \mathrm{m}$. The theoretical value of the overall spring constant for the non-perforated flat membrane is calculated as $\mathrm{k}_{\mathrm{z}}=0.34 \mathrm{~N} / \mathrm{m}$. Static structural analysis of the perforated step type switch is done using FEM $\mathrm{COMSOL}^{\mathrm{TM}}$ tool to find out its stiffness by applying a uniform force of $1.27 \mu \mathrm{N}$ on central actuation area for the deformation of $2 \mu \mathrm{m}$. The simulated value of $k_{z}$ is found to be $0.63 \mathrm{~N} / \mathrm{m}$. The deflection profile of the switch is shown in (Fig 4). For a maximum of $2 \mu \mathrm{m}$ deflection, switch undergoes a Von Mises stress of 58.2 MPa which is well under the gold yield strength as shown in (Fig 5).

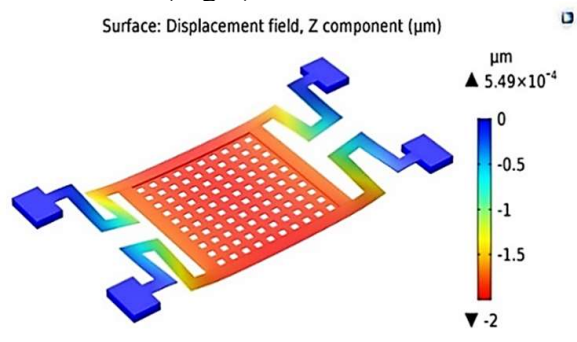

Figure 4: Deflection Profile of the Switch Using FEM COMSOL ${ }^{\mathrm{TM}}$

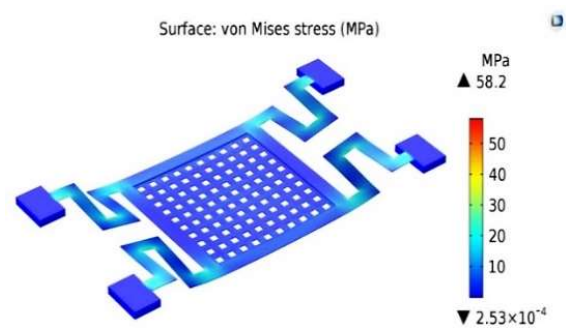

Figure 5: Von-Mises Stress Distribution in the Switch Using FEM COMSOL ${ }^{\mathrm{TM}}$

The electromechanical simulation was done using parametric voltage sweep of 0.5 Volt to 5.5 Volts between the upper and down electrode. Pull-in instability of the switch is found at $5.1 \mathrm{~V}$ for $1 / 3$ of the air gap displacement. The electromechanical simulation results for pull-in instability are shown in (Fig 6). 


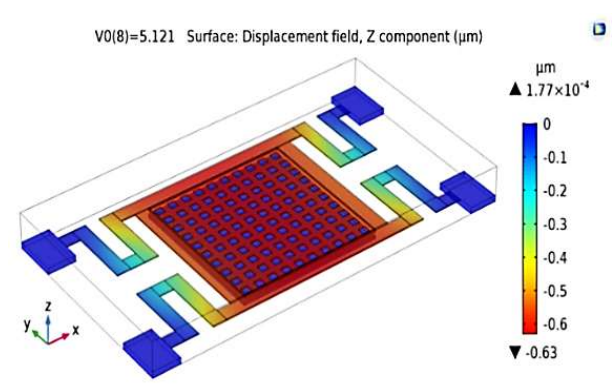

Figure 6: Electromechanical Simulation for Pull-in Instability

The switching time $\left(t_{s}\right)$ of the mechanical membrane of the switch decides the toggling time between the two states for which it makes and breaks the circuit in the transmission line. Mostly it depends on the applied bias voltage. A larger bias voltage $\left(V_{b}\right)$ than the pull-in voltage leads to a stronger electrostatic force to overcome mechanical restoring force at a faster rate. The switching time is given by Eq. (6) (Tilmans, 2002) as:

$$
t_{s} \cong 3.67 \frac{V_{P}}{V_{b} \omega_{0}}
$$

Table 2 shows the electromechanical simulation results for a pull-in voltage, mechanical resonant frequency as per the first mode of Eigenfrequency analysis and the switching time of the switch at $\quad V_{b}=2 V_{P}$ Volts with varying membrane thickness. (Fig 7) shows pull-in voltage instability for different membrane heights with an air gap of $2 \mu \mathrm{m}$.

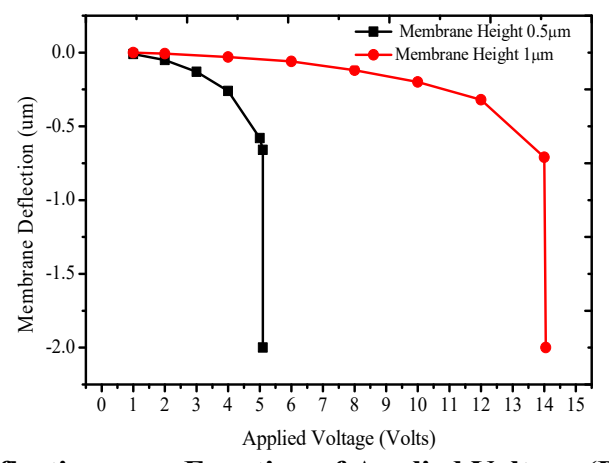

Figure 7: Membrane Deflections as a Function of Applied Voltage (Pull-in Voltage Analysis)

\begin{tabular}{|c|c|c|c|}
\hline $\begin{array}{c}\text { Membrane Thickness } \\
(\boldsymbol{\mu m})\end{array}$ & $\begin{array}{c}\text { Pull-in Voltage } \\
(\text { Volts })\end{array}$ & $\begin{array}{c}\text { Resonant Frequency } \boldsymbol{f}_{\boldsymbol{o}} \\
(\mathbf{K H z})\end{array}$ & $\begin{array}{c}\text { Switching Time } \\
\boldsymbol{t}_{\boldsymbol{s}}(\boldsymbol{\mu} \mathbf{s})\end{array}$ \\
\hline 0.5 & 5.1 & 11.42 & 25.57 \\
\hline 1 & 14 & 22.28 & 13.1 \\
\hline
\end{tabular}

Table 2: Electromechanical Simulation Results

RF Performance Analysis of the Proposed Switch

The electromagnetic behavior of the capacitive MEMS switch is governed by the capacitance ratio $\left(\mathrm{C}_{\mathrm{r}}\right)$ of the switch. The high capacitance ratio of down to upstate is desirable for good RF performance. The up- and downstate capacitance and the capacitance ratio is expressed in Eq. (7) to Eq. (9) (Rangra, 2005).

$$
\begin{aligned}
& C_{u p}=\frac{A \varepsilon_{0}}{g_{0}+\frac{t_{d}}{\varepsilon_{r}}}+C_{f} \\
& C_{d}=\frac{A \varepsilon_{0} \varepsilon_{r}}{t_{d}} \\
& C_{r}=\frac{C_{d}}{C_{u p}}=\frac{\varepsilon_{r} g_{0}}{t_{d}}
\end{aligned}
$$

Where ' $A$ ' is the capacitance overlap area between the upper and down electrode excluding perforation area, $\varepsilon_{0}$ is the free space permittivity, $g_{0}$ is the air gap between the upper electrode membrane and a dielectric layer in the up-state, $t_{d}$ is the thickness of the dielectric material, $\varepsilon_{r}$ is the dielectric constant of the insulating material, and $C_{f}$ is the fringing field capacitance. Eq. (9) Shows that, higher capacitance ratio can be obtained for the switches with a thinner dielectric material and high dielectric constants, and higher air gap results in the 
excellent RF performance. However, having the higher air gap tends to the higher actuation voltages, and thinner dielectric material affects the dielectric breakdown or charge injection problems which limit the reliability of the switch. Hence a proper trade-off between the air gap and dielectric materials are always preferred for better RF performances of the MEMS switches.

Another performance parameter of RF MEMS switch modeled as a two-port series RLC circuit is its simulated or measured scattering parameters. In ON-state $S_{11}$ and $S_{21}$ represent the return loss or reflection coefficient and the insertion loss while in OFF-state (actuated state) $S_{21}$ represent the isolation of the switch expressed by Eq. (10) and Eq. (11).

$S_{11}=-20 \log \left|\frac{-j \omega C_{u p} Z_{0}}{2+j \omega C_{u p} Z_{0}}\right|$

$S_{21}=-20 \log \left|\frac{2}{2+j \omega C_{d} Z_{0}}\right|$

The proposed $0.5 \mu \mathrm{m}$ thick gold metallic membrane switch with an air gap of $2 \mu \mathrm{m}$ and built on a CPW configuration with $0.3 \mu \mathrm{m}$ thick $\mathrm{Si}_{3} \mathrm{~N}_{4}$ as an insulating material $\left(\varepsilon_{\mathrm{r}}=7.6\right)$ is simulated in the up and down positions using the HFSS ${ }^{\mathrm{TM}}$ tool. Simulated RF performance of the proposed switch in the form of s-parameters for the frequency range of 1-60 GHz is shown in (Fig. 7) to (Fig 10).

In the up-state, insertion loss $S_{21}$ is better than $-0.5 \mathrm{~dB}$ for the frequency $37.5 \mathrm{GHz}$ and below, while it is less than $-1 \mathrm{~dB}$ for the subsets of $\mathrm{Ka}$ and $\mathrm{V}$-Band frequencies. Also return loss $\mathrm{S}_{11}$ during $\mathrm{ON}$-state is better than -10 $\mathrm{dB}$ for the frequency $38.5 \mathrm{GHz}$ and below. OFF-state simulation results show that switch has a maximum isolation of $-54.38 \mathrm{~dB}$ at $38.5 \mathrm{GHz}$ and better than $-20 \mathrm{~dB}$ for the frequencies in $\mathrm{Ka}$ and $\mathrm{V}$-Band subsets. Return loss in downstate is below $-0.11 \mathrm{~dB}$ in the $\mathrm{Ka}$ and $\mathrm{V}$-Band frequencies. From the simulated return loss plot in ON state, an upstate capacitance value $\left(C_{u}\right)$ is extracted using Eq. (10) for the frequency $f<<f_{0}$. and $S_{11}<<-10$ $\mathrm{dB}$. Similarly, the downstate capacitance value is extracted from isolation plots using Eq. (11) for the $S_{21}<<-10$ $\mathrm{dB}$ and $f \leq f_{0} / 4$. Simulated RF performance parameters are shown in Table 3 . The extracted value of the up capacitance shows a higher value than that of the calculated due to the fringing capacitance $\left(C_{f}\right)$. The extracted and the calculated values of the up- and down capacitance are in close percentage of validation.

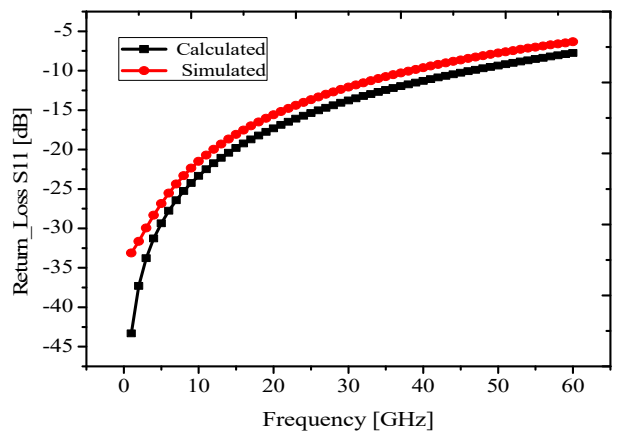

Figure 8: ON-State Return Loss S11 [dB]

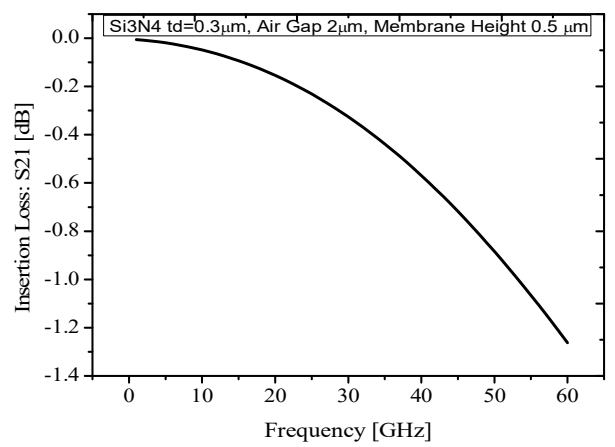

Figure 9: Simulated ON-State Insertion Loss S21 [dB] 


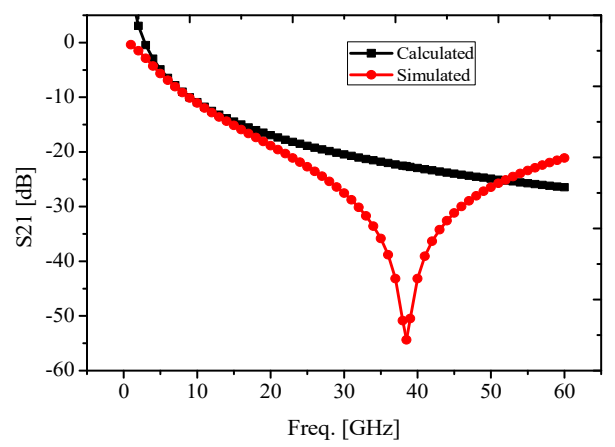

Figure. 10: OFF-State Isolation S21 [dB]

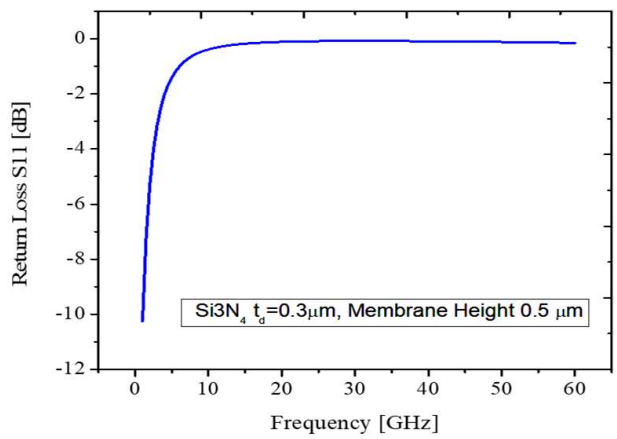

Figure 11: Simulated OFF-State Return Loss $S_{11}[\mathrm{~dB}]$

\begin{tabular}{|c|c|c|}
\hline Performance Metric & Calculated Value & Extracted Value \\
\hline Up-state capacitance $C_{u p}(f F)$ & 43.41 & 52.68 \\
\hline Down-state capacitance $C_{d}(p F)$ & 2.24 & 2.27 \\
\hline Capacitance ratio $\left(C_{d} / C_{u p}\right)$ & 52 & 43 \\
\hline
\end{tabular}

Table 3: Calculated and Extracted RF Performance Parameters

Impact of Variations of Geometric Parameter Trade Off's:

In this section geometric parameters such as membrane height, dielectric height and air gap using different dielectric material like $\mathrm{Si}_{3} \mathrm{~N}_{4}$ and $\mathrm{HfO}_{2}$ are varied to investigate $\mathrm{ON}$ - and OFF-state RF performance of the switch.

\section{Up-State Response}

As seen from Eq. (7), the upstate capacitance is dependent on actuation area, air gap, and thickness of the dielectric and dielectric constant. For the proposed switch, the area of the actuation is constant while dielectric material, dielectric thickness, and air gap are varied to see the impact on RF performance. An increase in the air gap decreases the capacitance value, which improves the switch RF performance. However, increases in dielectric thickness and membrane height do not show a significant change in RF performance in each case of the air gap. ON state RF performance of the switch with geometric trade-off's is shown in (Fig 11) to (Fig 13).

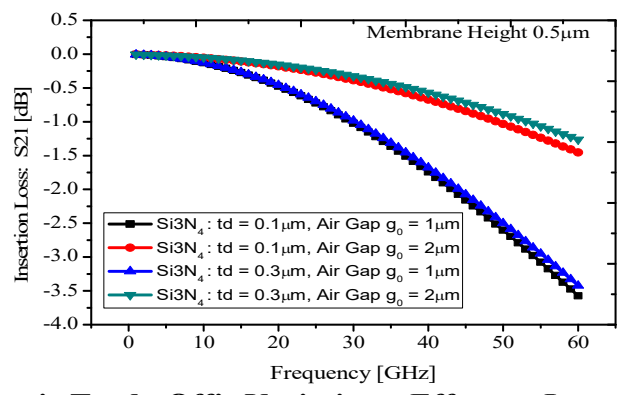

Figure 12: Geometric Trade-Off's Variations: Effect on Insertion Loss $S_{21}$ [dB] 


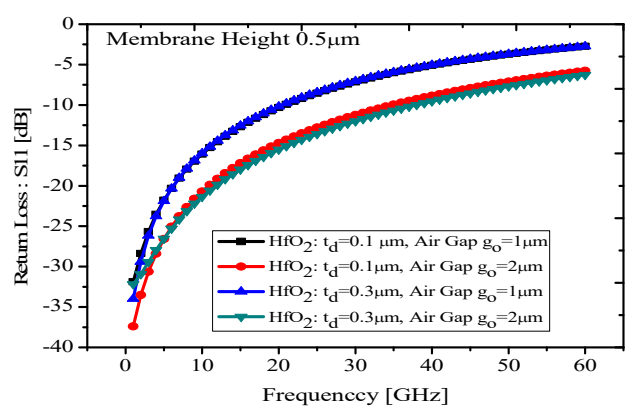

Figure 13: Geometric Trade-Off's Variations: Effect on Return Loss $\mathrm{S}_{11}$ [dB]

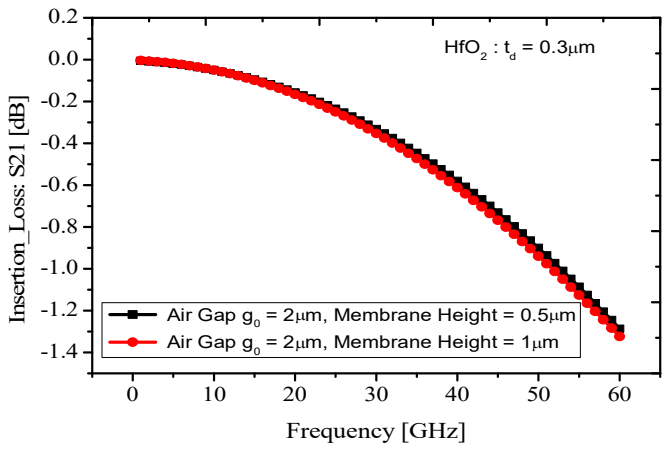

Figure 14: Effect of Membrane Height on Insertion Loss

\section{Down-State Response}

Eq. (8) shows that downstate capacitance is directly proportional to the dielectric constant of the insulator material while inversely proportional to insulator height. (Fig 14) to (Fig 16) shows downstate RF performance for geometric parameter variations. RF performance shows that thinner dielectric material gives better isolation in lower frequency ranges while thicker insulating material improves performance in higher frequency ranges. As compared to $\mathrm{Si}_{3} \mathrm{~N}_{4}$ as a dielectric, $\mathrm{HfO}_{2}$ due to its high-k gives the highest value of isolation at the resonant frequencies. Effect of membrane height does not show a significant change in isolation for varying dielectric heights.

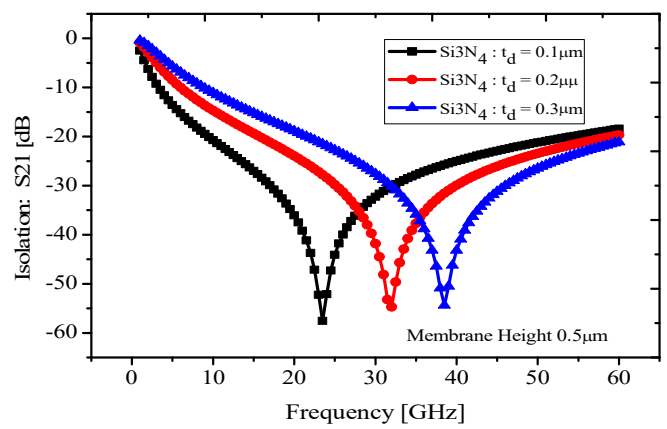

Figure 15: Effect of the Geometric Trade-Off's Variations on Isolation $\mathrm{S}_{21}[\mathrm{~dB}]$ with $\mathrm{Si}_{3} \mathrm{~N}_{4}$ Dielectric

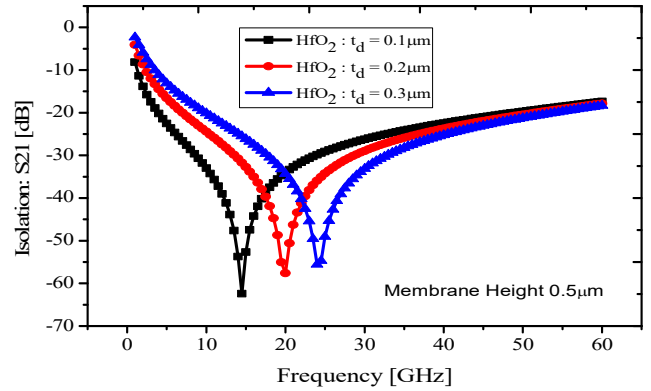

Figure 16: Effect of the Geometric Trade-Off's Variations on Isolation $\mathrm{S}_{21}[\mathrm{~dB}]$ with $\mathrm{HfO}_{2}$ Dielectric 


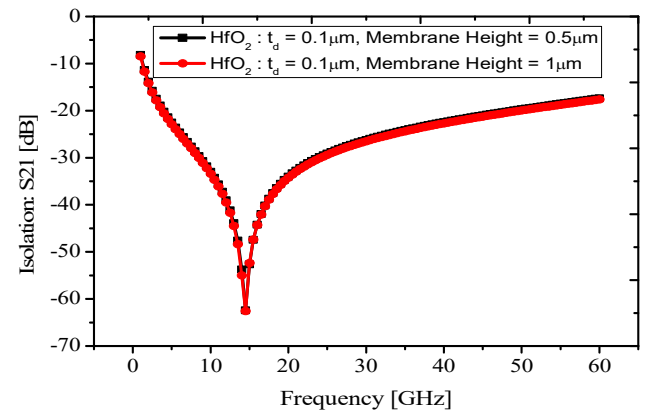

Figure 17: Effect of Membrane Height on Isolation

Simulation results for varying geometric parameters in the frequency range of $1 \mathrm{GHz}$ to $60 \mathrm{GHz}$ as shown in (Fig 11) to (Fig 16) show that variations of the geometric parameters like an air gap, dielectric thickness, and membrane thickness and use of the different dielectric materials with low- and high-k values, switch RF performance can be optimized for various frequency ranges in subsets of Ka- and V-band. Thinner membrane heights results in a low pull-in voltage than thicker membranes, however, low pull-in voltage tends to slow switching speeds of the devices. With $\mathrm{Si}_{3} \mathrm{~N}_{4}$ as a dielectric material, switch RF performance improves in higher frequency bands with an increase of dielectric thickness while the use of high-k dielectric material gives better performance in low-frequency bands.

\section{Conclusions}

The RF MEMS switch is designed and simulated for the subsets of Ka and V-Band applications using $\mathrm{Si}_{3} \mathrm{~N}_{4}$ and $\mathrm{HfO}_{2}$ dielectrics. A novel approach of a step-in nature actuation electrode is used to ensure low-pull in voltage and uniform contact of the upper electrode to dielectric layer on the down electrode. Use of the ribs surrounding to the steps of the switch results in low Von-mises stress during the actuation of the switch. With $0.3 \mu \mathrm{m}$ thick Si3N4 insulating material and $0.5 \mu \mathrm{m}$ membrane height, switch optimized the RF characteristics in subsets of $\mathrm{Ka}$ - and $\mathrm{V}$-Band frequency ranges. The on-state RF characteristics shows insertion loss of better than $-0.5 \mathrm{~dB}$ and return loss of better than $\quad-10 \mathrm{~dB}$ below $37.5 \mathrm{GHz}$. The off-state RF performance shows the maximum isolation of $\quad-54.38 \mathrm{~dB}$ at $38.5 \mathrm{GHz}$. Varying geometric parameters like an air gap, dielectric height, and the membrane thickness affects the electromagnetic and electromechanical performance of the switch.

\section{References}

1. G.M. Rebeiz. 2003. RF MEMS: Theory, Design, and Technology. Wiley India Edition, John Wiley \& Sons, Inc. Hoboken, NJ, USA, (2003), ISBN: 978-81-265-2580-5.

2. H.A.C. Tilmans. 2002. MEMS Components for wireless communications (invited paper). In Proc. of the Eurosensors XVI The 16th European Conference on Solid-State Transducers, Prague, Czech Republic, 15-18 September 2002.

3. M. Angira, and K. Rangra. 2015. Design and investigation of a low insertion loss, broadband, enhanced self and hold down power RF-MEMS switch. Microsystem Technologies, Vol.21, No.6, pp.1173-1178.

4. A.K. Sharma, and N. Gupta. 2012. Material Selection of RF-MEMS switch used for reconfigurable antenna using Ashby's Methodology. Progress in Electromagnetics Research Letters, Vol. 31, pp. 147-157.

5. P. Bahamanyar, K. Mafinezad, and M. Bahamanyar. 2010. Switching performance analysis in RF MEMS Capacitive shunt switches by geometric parameters trade-offs. Circuits and Systems (APCCAS), 2010 IEEE Asia Pacific Conference, pp.831-834.

6. Y. Mafinejad, A.Z. Kouzani, K. Mafinezhad, and D. Izadi. 2009. Design and Simulation of a RF MEMS Shunt Switch for Ka and V Bands and the Impact of Varying Its Geometrical Parameters. Circuits and Systems, MWSCAS '09. 52nd IEEE International Midwest Symposium, Mexico, 2009, pp.823-26.

7. J.M.Rebeiz, and J.B.Muldavin. 2001. RF MEMS Switches and Switch Circuits. IEEE Microwave Magazine, Vol.2, No.4, pp. 59-71.

8. K.J Rangra. 2005. Electrostatic Low Actuation Voltage RF MEMS switches for Telecommunications. Ph.D. Thesis, University of Trento, Italy. 